\title{
S1. JSBACH4-FF
}

Simulations were conducted with the git feature/forest branch (commit 77a88be0) of JSBACH4 (based on jsbach4.20p7) in standalone mode hosted within the MPI-ESM environment. We used the MPI-ESM environment revision 9585 of the mpiesmlandveg branch, which is the development branch of the department "The Land in the Earth System" of the Max Planck Institute for Meteorology.

The JSBACH4-FF code and information on how to repeat the runs is archived together with other supplementary information by the Max Planck Institute for Meteorology (https://pure.mpg.de/pubman/faces/ViewItemFullPage.jsp?itemId=item_ 3032727) and can be obtained by contacting publications@mpimet.mpg.de.

\section{S2. Derivation of harvest maps}

From the derived age maps based on Poulter et al. (2018), we further derived harvest maps for each simulation year, such that the simulated age distribution conformed with the observed one in 2010. We assumed that the fractions given by Poulter et al. (2018) are equally distributed over the ten years covered by each age-class:

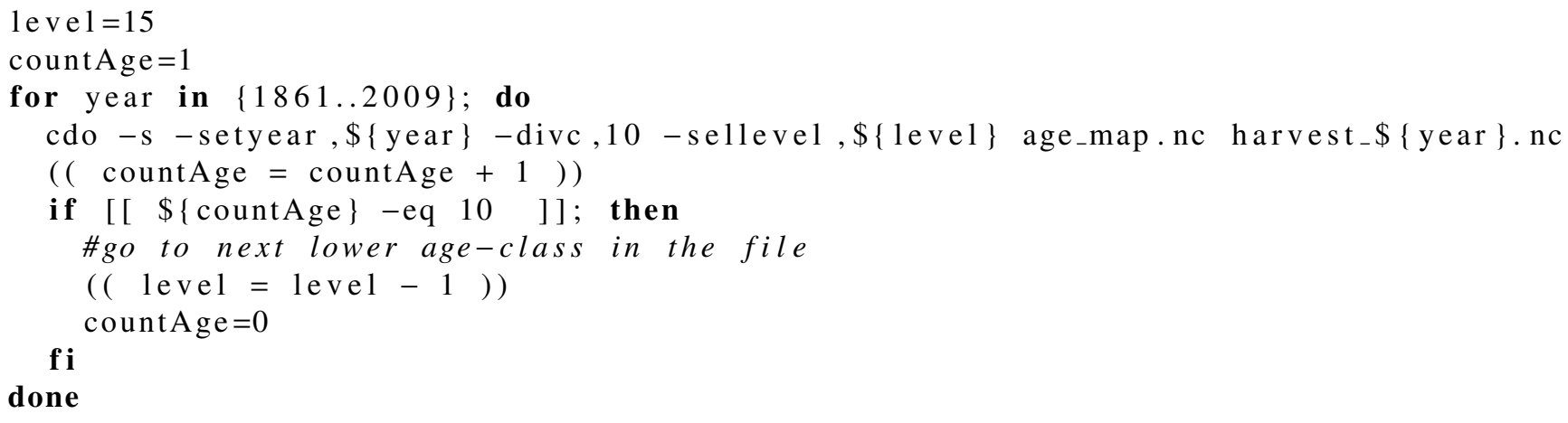

where level corresponds to the age-class in the map by Poulter et al. (2018).

\section{S3. NRSME}

A max-min normalised root mean squared error (NRSME $\mathrm{Nax}_{\mathrm{Min}}$ ) was calculated as described in Section 2.4.5 in the main text. Figure S3.1 shows the NRMSE $\mathrm{Max}_{\text {-Min }}$ per age-class for each simulation, variable and region.

\section{S4. Maps}

Figures S4.2-S4.4 show maps of the observation-based data for GPP, LAI and AGB scaled to JSBACH's forested area (see Section 2.4.1, main text), as well as different kinds of difference maps.

\section{References}

Poulter, B., Aragão, L., Andela, N., Bellassen, V., Ciais, P., Kato, T., Lin, X., Nachin, B., Luyssaert, S., Pederson, N., Peylin, P., Piao, S., Saatchi, S., Schepaschenko, D., Schelhaas, M., and Shivdenko, A.: The global forest age dataset (GFADv1.0), link to NetCDF file, https://doi.org/10.1594/PANGAEA.889943, URL https: //doi.org/10.1594/PANGAEA.889943, 2018. 

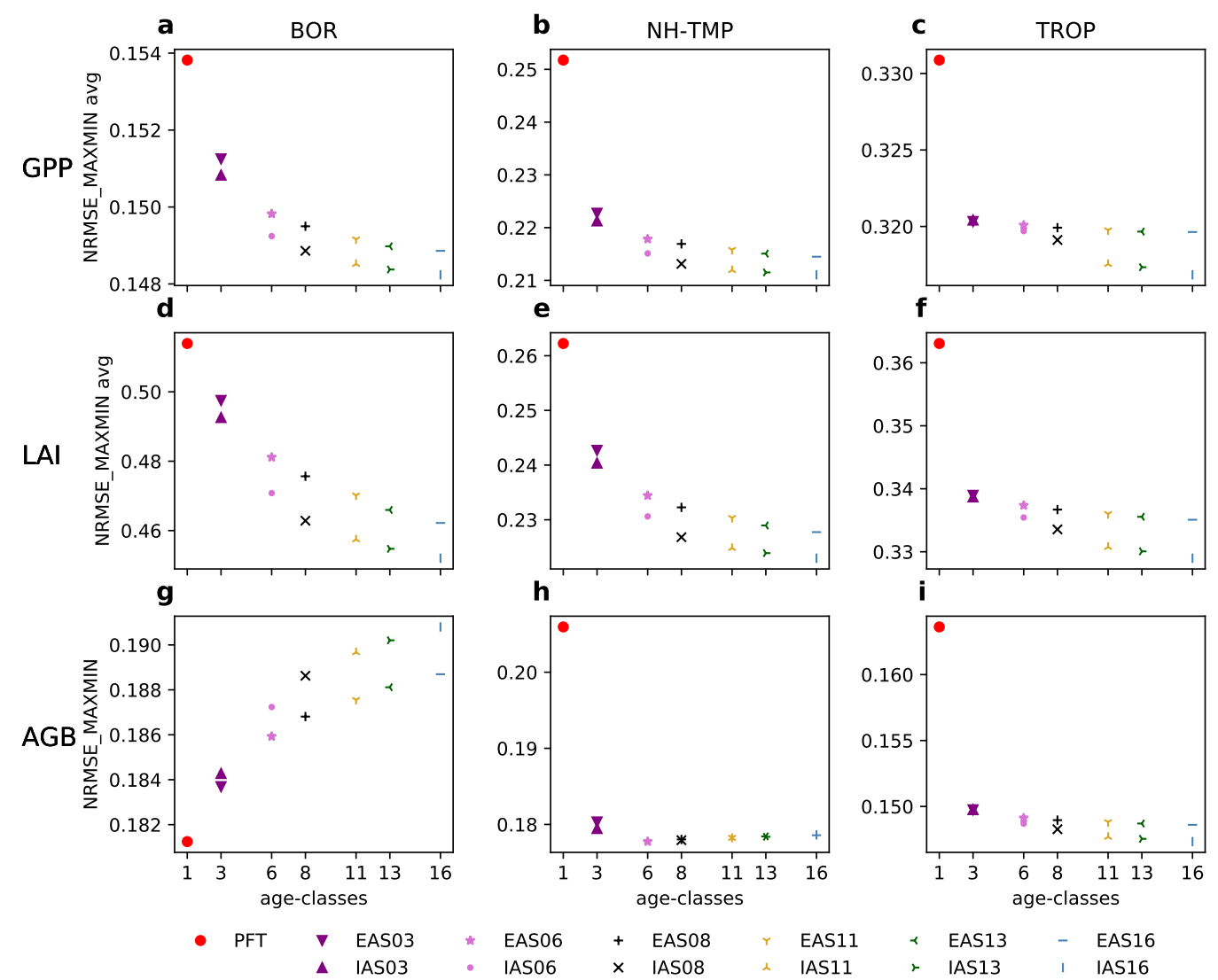

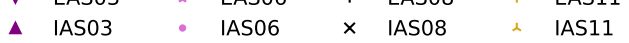

\begin{tabular}{|c|c|}
\hline EAS13 & - \\
\hline IAS13 & I \\
\hline
\end{tabular}

Fig. S3.1: Change in NRMSE Max-Min (see Section 2.4.5 and Fig. 5, main text) when increasing the number of age-classes. For gross primary production (GPP) and leaf area index (LAI), an averaging has been conducted giving equal weights to each of the four seasons (panel a to f). 

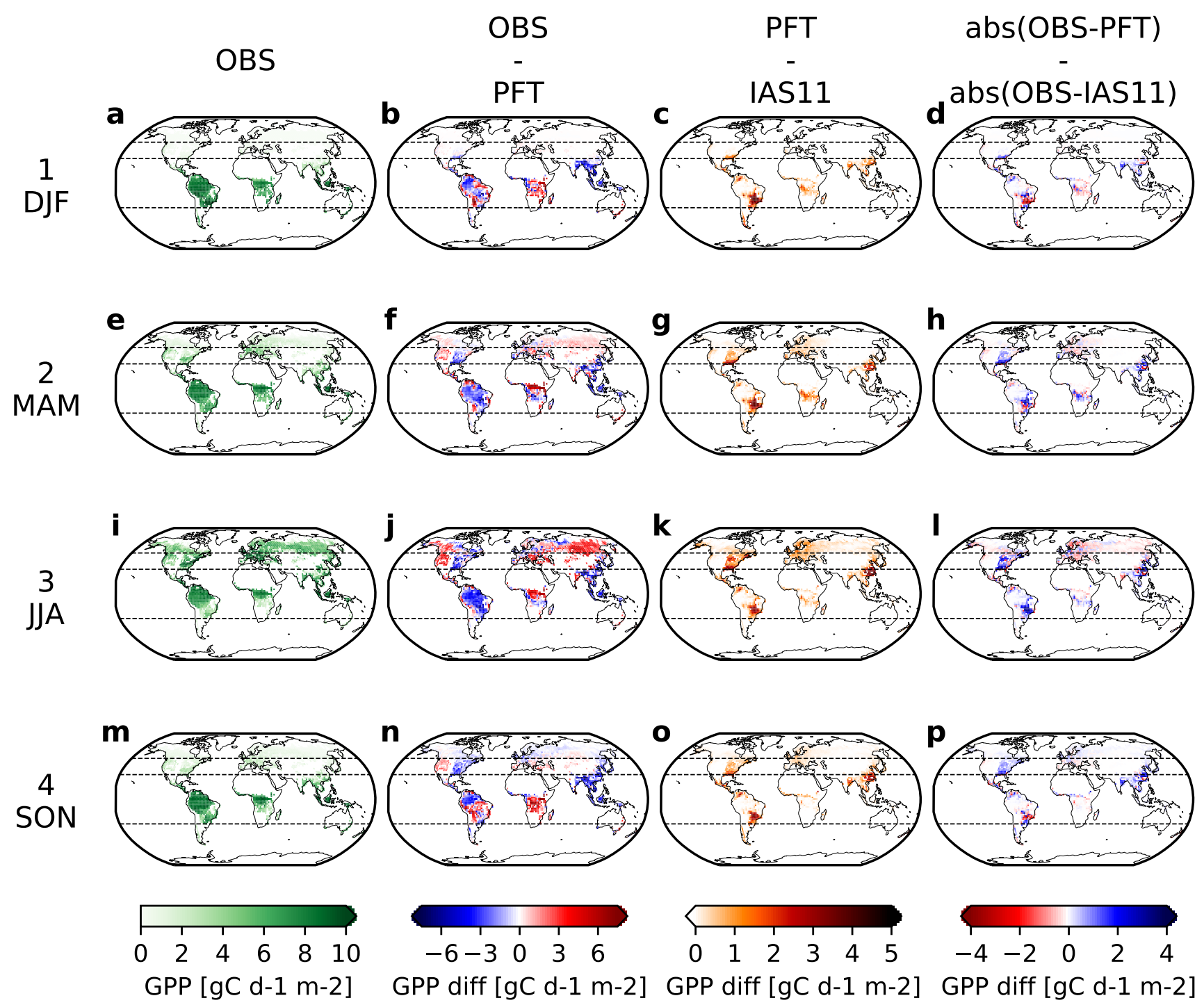

Fig. S4.2: Observation-based data scaled to JSBACH's forested area (see Section 2.4.1, main text) and difference maps for gross primary production (GPP). Rows show the four seasons. The first column shows the observation-based data (OBS). The second column shows the difference between OBS and the 2001-2010 average of the PFT simulation results. The third column shows the difference between OBS and IAS11 and OBS and PFT simulation results (see Table 1, main text for simulation names). The difference between the differences to the observation-based data equals the difference between the simulation results ((OBS-IAS11)-(OBSPFT) $=$ PFT-IAS11). The fourth and last column also shows the difference, however, from the absolute differences (abs(OBS-PFT)-abs(OBS-IAS11)), i.e. it shows where the results from the simulation with age-classes deviate more (red) or less (blue) from the observation-based data than the PFT simulation results. Dashed lines mark the three selected regions - boreal, northern hemisphere temperate, and tropical (see Table 2, main text). 

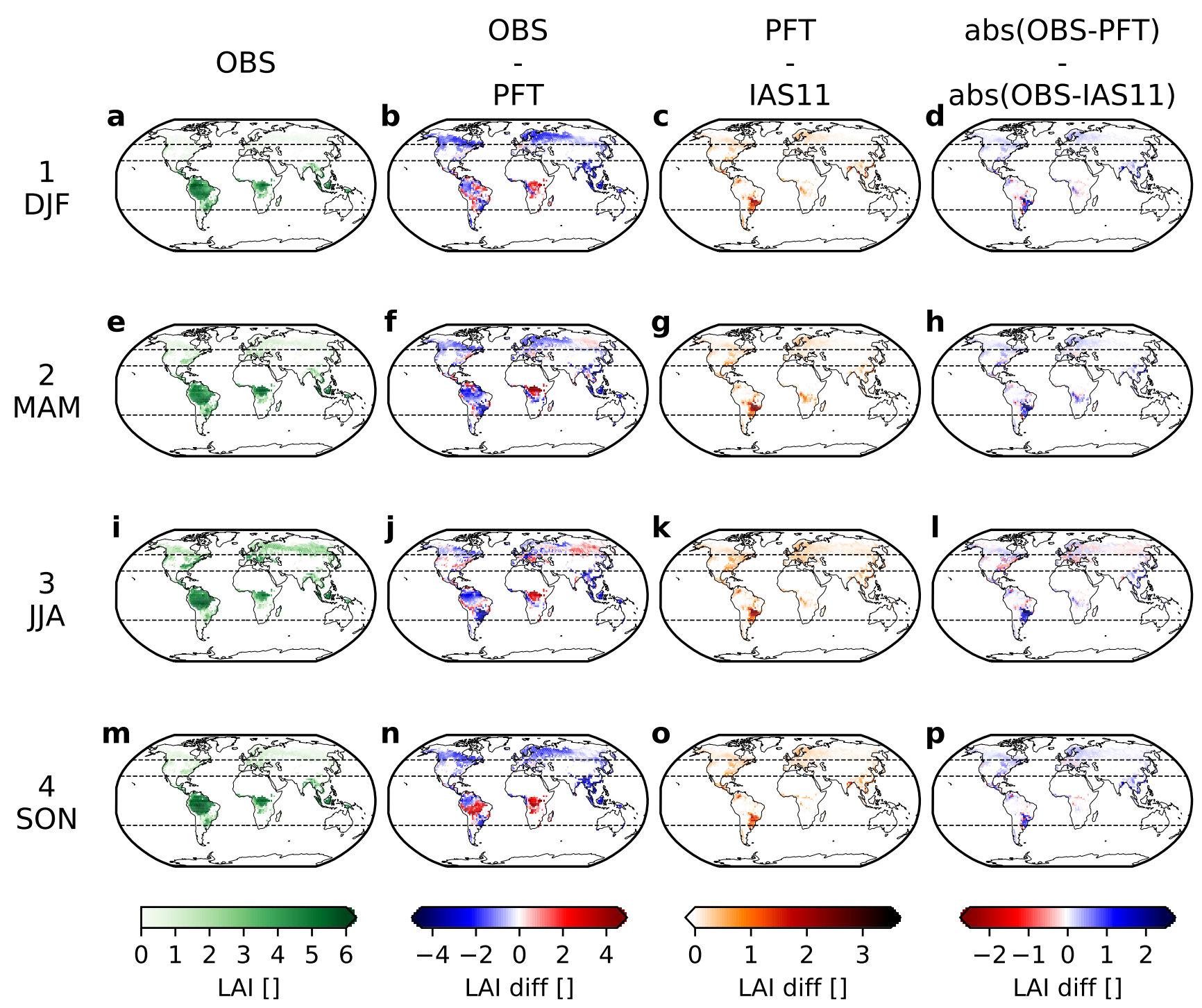

Fig. S4.3: Same as Fig. S4.2, here for leaf area index (LAI).

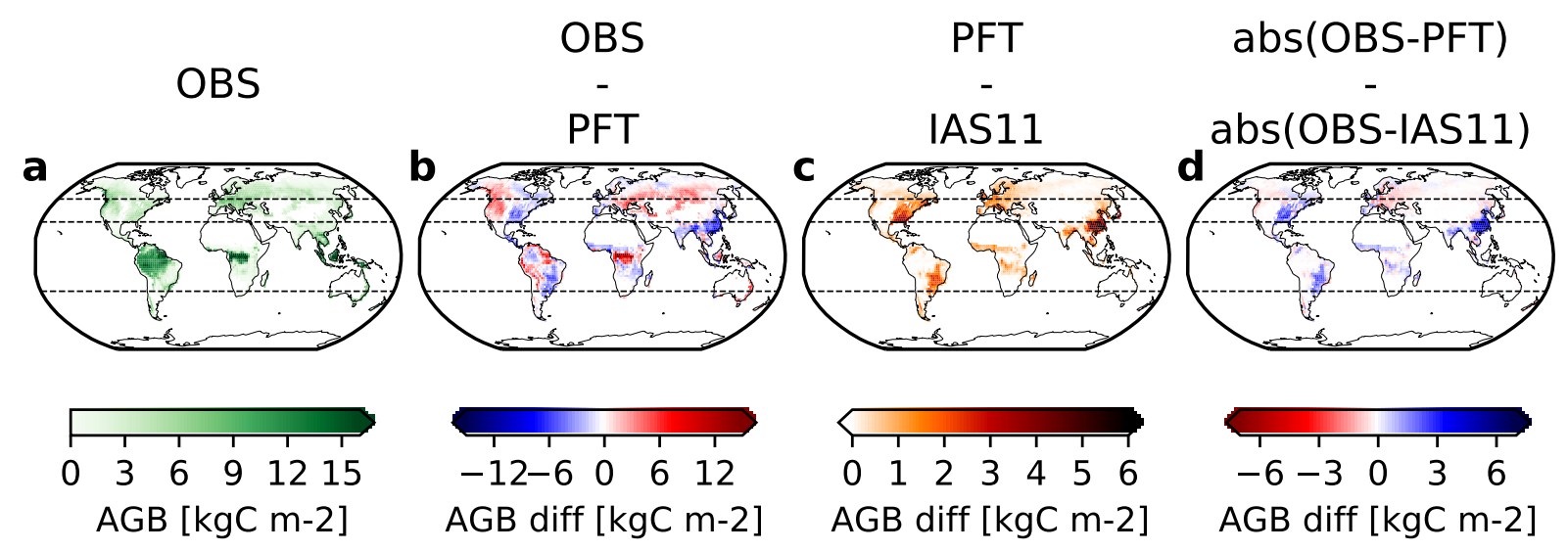

Fig. S4.4: Same as Fig. S4.2, here for above ground-biomass (AGB). 